\title{
New Perspectives on the Recurrent BRCA Mutations and Clinical Variability
}

\author{
Ibrahim SAHIN ${ }^{1}$, Hanife SAAT ${ }^{1}$, Sercan AKSOY ${ }^{2}$ \\ ${ }^{1}$ University of Health Sciences, Dışkapı Yıldırım Beyazıt Training and Research Hospital, Department of Medical Genetics \\ ${ }^{2}$ Hacettepe University, Faculty of Medicine, Department of Medical Oncology, Ankara, TURKEY
}

\begin{abstract}
Familial cancer syndromes include about 5\% to 10\% of all tumors and are attributable to genetic defects. Hereditary breast and ovarian cancer syndrome (HBOC) is distinguished by an elevated risk for male and female breast cancer, ovarian cancer (Fallopian tube and primary peritoneal cancers), and to a smaller degree, other cancers such as prostate cancer, pancreatic cancer, and melanoma. In this study, we performed next-generation sequencing or a comprehensive cancer panel on 335 patients to explain the importance of recurrent germline mutations of BRCA1-2 and their clinical results and determine how often BRCA gene mutations were seen in people suspected of Lynch syndrome. BRCA2 mutations were more predominant ( $n=212,63.3 \%$ ) than BRCA1 mutations (123, 36.7\%) in all groups except for the ovarian and breast-ovarian groups. The most common recurrent mutations were $B R C A 2: C .3318 C>G$, BRCA2:c.632-2A > C, BRCA1:c.5266dupC, BRCA2:C.9317G > A, BRCA2:C.8452G > A, BRCA1:c.2800C > T, respectively. BRCA1 variants were associated with ovarian cancers, and BRCA2 variants show a higher incidence of extra-ovarian malignancy. The principal finding of our study is that BRCA2 mutations were more predominant not just in the breast group but also in the colorectal group. This is the first study mentioning the importance of BRCA2 variants in patients with Lynch Syndrome (LS) phenotype to the best of our knowledge. Our data broadens the spectrum of BRCA gene mutations and provides insights for genotype-phenotype correlations for familial cancer syndromes.
\end{abstract}

Keywords: Hereditary breast and ovarian cancer syndrome, Lynch Syndrome, BRCA1, BRCA2

\section{INTRODUCTION}

Familial cancer syndromes include about 5\% to $10 \%$ of all tumors and are attributable to genetic defects. ${ }^{1}$ Hereditary breast and ovarian cancer syndrome (HBOC), Li-Fraumeni syndrome, Cowden syndrome, MUTYH-associated polyposis (MAP), and Lynch syndrome are examples of inherited cancer syndromes. ${ }^{2}$ HBOC is distinguished by an elevated risk for male and female breast cancer, ovarian cancer (Fallopian tube and primary peritoneal cancers), and to a smaller degree, other cancers such as prostate cancer, pancreatic cancer, and melanoma. Li-Fraumeni syndrome, Cowden syndrome, Lynch syndrome, and ATM, PALB2,
CHEK2 gene mutations could result in a similar phenotype with HBOC. ${ }^{3}$

Germline genetic testing for risk evaluation of inherited cancers has been standard for breast and ovarian cancers. Heritable breast cancers account for 5 to $10 \%$ of all breast cancers, and monogenic, extremely penetrant genes trigger them. BRCA1 and BRCA2 germline variants account for up to $30 \%$ of all inheritable breast cancers. ${ }^{4}$ There are more than 2000 documented pathogenic variants in the BRCA1 and BRCA2 genes. About 2.9\% of all people receiving molecular genetic tests for BRCA1 and BRCA2 would have an unknown clinical significance variant. ${ }^{5}$ 
The most prevalent malignancy in individuals with a germline BRCA1 or BRCA2 pathogenic variant is breast cancer, which varies from $46 \%$ to $87 \%$. BRCA germline pathogenic variants raise the incidence of ovarian cancer from $16.5 \%$ to $63 \%$. BRCA1 and BRCA2 germline pathogenic forms are inherited in an autosomal dominant pattern. Each person with a BRCA1 or BRCA2 germline pathogenic variant has a $50 \%$ risk of transmitting the variant to their offspring. Around $90 \%$ of pathogenic variants in BRCA1 and BRCA2 are observed using gene sequencing, with another $10 \%$ identified through gene duplication/deletion analysis, which differs across various communities. ${ }^{6}$ The effects of BRCA mutations have also been discovered to be connected to prostate, pancreatic, stomach, and colorectal cancers, although it is difficult to determine the level of harm. ${ }^{7}$

The BRCA tumor suppressor genes are active in homologous recombination. BRCA1 is correlated with various pathways that include cellular pathway components, such as cell-cycle progression, gene transcription control, DNA damage response, and ubiquitination. BRCA1 is observed to colocalize with BRCA2 and RAD51 at sites of DNA damage and is recruited to sites of DNA damage through the association with RAD51 and then enables RAD51-mediated homologous recombination repair of DNA double-strand breaks. ${ }^{8}$ Therefore, BRCA defective cells are incredibly susceptible to DNA damage and cancer development. ${ }^{9}$ PARP enzymes are involved in the process of detecting and repairing DNA damage. ${ }^{10}$ The PARP1 inhibitors allow these double-strand breaks to develop, and in BRCA mutation-positive cancers, they are not effectively repaired, leading to the death of cells. ${ }^{11}$

More frequently observed in women over the age of 40s, ovarian cancer frequency increases in developed countries. Ovarian cancer is the most deadly in female reproductive cancers. ${ }^{12}$ Pancreatic cancer is one of the deadliest cancers in both the United States and Europe, and fourth in the world in causes of cancer death. In those diagnosed with pancreatic cancer, a family history of the disease is observed in $5-10 \%$ of the cases. One of the more common types of pancreatic ductal adenocarcinoma appears in families with ovary or breast cancer. ${ }^{13}$ BRCA1 and BRCA2 mutations also are associated with an elevated incidence of pancreatic cancer. ${ }^{14}$
In this study, we performed next-generation sequencing or a comprehensive cancer panel to explain the importance of recurrent germline mutations of BRCA1-2 and their clinical results and determine how often BRCA gene mutations were seen in people suspected of Lynch syndrome. Our data broadens the spectrum of BRCA gene mutations and provides insights for genotype-phenotype correlations for familial cancer syndromes.

\section{MATERIALS AND METHODS}

\section{Patients}

The Ethics Committee of the University of Health Sciences, Dışkapı Yıldırım Beyazıt Training and Research Hospital approved the study (08.03.2021, 106/27). Consent for the publication and any additional related information was taken from the 335 patients or their parents involved in the study. Most of the patients came to our clinic in their first few years of diagnosis. Clinical histories and molecular results were reviewed for all patients who examined at the Department of Medical Oncology, Hacettepe University, Faculty of Medicine and Department of Medical Genetics, University of Health Sciences, Dışkapı Yıldırım Beyazıt Training and Research Hospital, Ankara, Turkey. Patients underwent BRCA1-2 or comprehensive cancer panel test between January 2017 and December 2020 at Ankara Central Genetic Laboratory (Ankara, Turkey). Patients were evaluated according to National Comprehensive Cancer Network (NCCN) guidelines for breast-ovarian and Lynch syndrome. All have a strong family history with at least three cancers in relatives (1st, 2nd, 3rd degree). Patients with uncertain/missing data have been filtered.

Participants were divided into seven groups, based on each participant's personal history. First group is breast $(n=249,74.3 \%)$, second group is family $(n=34,10 \%)$, third group is ovarian $(n=22$, $6.5 \%)$, fourth group is breast-ovarian $(\mathrm{n}=18$, $5.3 \%)$, fifth group is breast-colorectal $(\mathrm{n}=7,2 \%)$, sixth group is pancreas $(n=4,1.2 \%)$ and seventh group is prostate $(1,0.3 \%)$. In the second group (family), patients with unspecific cancers (endometrium, bladder, gallbladder, testicular, melanoma, leukemia-lymphoma, liver, lung, larynx) have been chosen. In the second and fifth groups, most 
of the patients were suspected of Lynch syndrome (LS). In patients suspected of LS, a comprehensive cancer panel has been performed. Other patients underwent BRCA1-2 sequencing and MLPA tests. Participants positive for either the BRCA1 or the BRCA2 mutations have been chosen for the study.

\section{DNA Panels and NGS}

Blood samples were collected in EDTA tubes. The patients' DNA was extracted according to the manufacturer's standard procedure using the QIAamp DNA Blood Midi Kit (Qiagen, Hilden, Germany) by QIAcube (Qiagen Inc Mississauga, ON, Canada). The DNA samples were quantified with a NanoDrop 1000 (Thermo Fisher Scientific Inc., MA, USA) spectrophotometer.

Qiaseq targeted DNA panel (DHS-102Z, Human BRCA1 and BRCA2 Panel) or Multiplicom BRCA MASTR Dx (Multiplicom N.V., Niel, Belgium) kits have been used for BRCA sequencing. Two different multigene panels have been used depending on the dates. From 2017 to 2018, Qiagen Qiaseq Hereditary Custom Cancer Panel (Qiagen, Hilden, Germany) has been used, and from 2018 to 2021, Sophia Hereditary Cancer Solution Panel (Sophia Genetics, Saint-Sulp) has been used in the center. The sequencing was performed on the Illumina MiSeq system (Illumina Inc., San Diego, CA, USA). The data were analyzed on QIAGEN Clinical Insight (QCI ${ }^{\mathrm{TM}}$ ) Analyze software (QIAGEN, Hilden, Germany) or Sophia DDM software v4 (Sophia Genetics, Saint-Sulp). Visualization of the data was performed with IGV 2.7.2 (Broad Institute) software.

For MLPA confirmation, SALSA MLPA probemix P002 BRCA1, SALSA MLPA probemix P087 BRCA1, SALSA MLPA probemix P045 BRCA2/ CHEK2, SALSA MLPA probemix P077 BRCA2 (MRC Holland, Amsterdam, Netherlands) kits were performed.

In hereditary cancer syndrome families, only one patient with a strong clinical story has been tested, and other family members have been validated with the known mutation with Sanger sequencing. If the mutation was VUS supporting clinical phenotype and no other mutation was responsible, then one more family member with cancer was also studied with the panel to ensure the causal gene and mutation. With methods like capillary sequencing, all identified variants were verified. Genetic testing is recommended for at-risk family members.

\section{Interpretations, Descriptive Statistics and Graphics}

Thorough evaluation and analysis of available evidence (e.g., population frequency information, reported case notes, case/control and functional tests, internal co-occurrence and segregation data, evolutionary conservation, and in silico predictions) were carried out on all variants, except for previously characterized benign alterations. In compliance with the recommendations issued by the American College of Medical Genetics and Genomics and the Association for Molecular Pathology, variants were categorized as pathogenic, likely pathogenic, variant of unknown significance (VUS), likely benign, and benign. ${ }^{15}$ Pathogenic, likely pathogenic, and strong VUS (supports clinical phenotype and no other mutation responsible detected) variations have been included in the study. Descriptive statistical calculations have been made, and graphics have been prepared with Python 3.9.2 (IPython 7.19.0).

\section{RESULTS}

Females $(315,94 \%)$ were more than males $(20$, $6 \%$ ). The mean age was 48 (in females 48 and males 52) with a minimum age of 18 and a maximum of 83. Most of the patients were between 40-60 ages (Figure 1). All the participants were positive for either the BRCA1 or the BRCA2 mutations.

Three hundred thirty-three mutations were heterozygous (99.4\%), and only two were homozygous (0.6\%). BRCA2:c.6842G $>\mathrm{A}$ and BRCA2:c.7559G $>$ C were homozygous. BRCA2 mutations were more predominant $(n=212,63.3 \%)$ than BRCA1 mutations $(123,36.7 \%)$ in all groups except for the ovarian and breast-ovarian groups (Figure 2). BRCA2:c.3318C > G, BRCA2:c.6322A > C, BRCA1:c.5266dupC, BRCA2:c.9317G>A recurrent mutations were predominant in only the breast group. BRCA1:c.181T > G and 


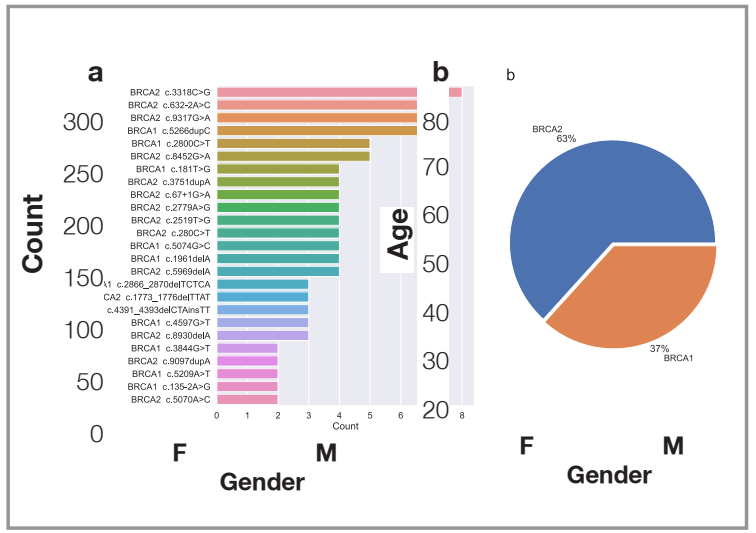

Figure 1. Patients' characteristics

a) Bar plot showing the number of patients in terms of gender b) Boxplot showing mean (green triangle) and median (black line) age of the patients in terms of gender

\section{DISCUSSION}

The principal finding of our study is that BRCA2 mutations were more predominant not just in the breast group but also in colorectal and pancreas groups. Patients with LS phenotype were carrying BRCA2 mutations.

The mean age in males and females were 52 and 48 , respectively. Most of the cancers observed between 40-50 years of age. There were two patients below 20 years of age, and they were carrying BRCA2 mutations. Since the minimum age was 18 , genetic counseling should be given carefully.

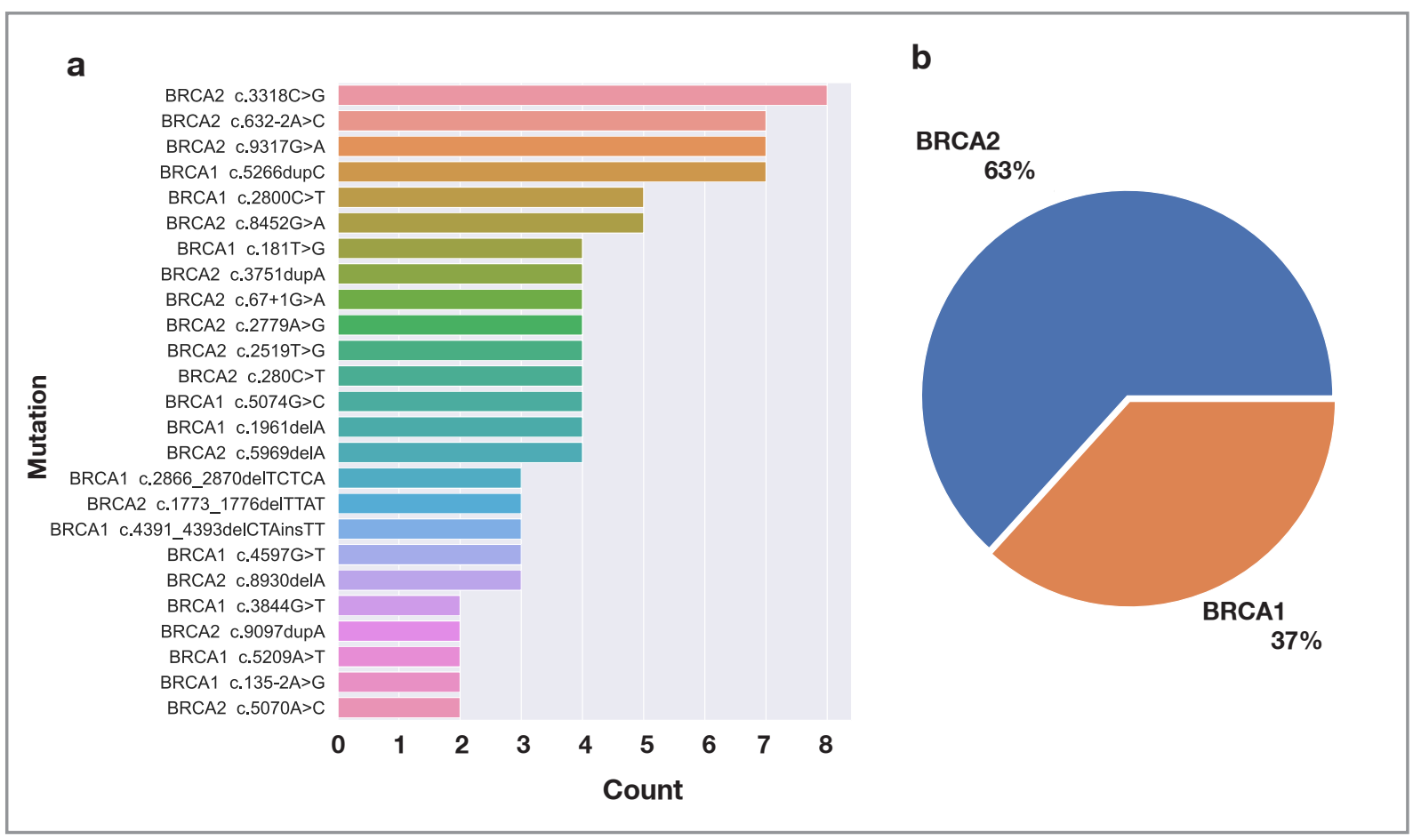

Figure 2. Spectrum of the genes and mutations

a) Bar plot showing the most common mutations in the study. b) Pie chart showing the spectrum of the mutated genes with percentages in the study.

BRCA1:c.3844G > T mutations were observed in the breast-ovarian group more than once.

A detected mutation was validated through Sanger sequencing in all other family members with cancer. BRCA1 variants were associated with ovarian cancers, and BRCA2 variants show a higher incidence of extra ovarian malignancy.
This is the first study mentioning the importance of BRCA2 variants in patients with Lynch Syndrome (LS) phenotype to the best of our knowledge. Forty-one of the patients were referred to our clinic with LS phenotype, and 29 of them were carrying BRCA2 mutations. Oh, et al., reported an increased risk of colorectal cancer in BRCA1 mutation carriers but not BRCA2. ${ }^{16}$ However, our study provides evidence of increased risk in colorectal 
cancer, particularly in BRCA2 mutation carriers. BRCA1 mutations were associated with ovarian and breast-ovarian phenotype, but BRCA2 mutations were associated with extra ovarian cancers, especially colorectal, pancreas, prostate, and other cancers. Recurrent mutations were observed only in the breast and ovarian group, not in other groups.

Knowing if one has a BRCA mutation provides the potential to improve cancer detection and increase follow-up procedures. Several recently published therapies, specifically the PARP inhibitors, help advanced breast cancer patients. ${ }^{17}$ Prophylactic (and conservative) interventions such as mastectomy and oophorectomy, genetic counseling, and drug management have been recommended by the US National Comprehensive Cancer Network (NCCN) Genetic/Familial High-Risk Assessment: Breast and Ovarian guidelines, focusing on those that have been identified as BRCA carriers. ${ }^{18}$

Researchers have also shown that BRCA1 gene mutations occur more often in high-risk cancer databases than BRCA2 gene mutations. ${ }^{19}$ One research has concluded that mutations in the BRCA2 gene are more numerous than those in the BRCA1 gene. ${ }^{20}$ Interestingly, we detected more BRCA2related variants than BRCA1 variants in our patients. BRCA2 mutations were common both in males and females. Even though increased risks have been discussed with very frequently observed polymorphic variant BRCA2 truncating allele c.9976A > T (p.Lys3326Ter), it has been reported benign many times, and functionally no difference has been found when compared with normal protein, we have filtered the variant from our study. ${ }^{21}$

The BRCA1:c.5382insC is common in Europe. Widespread BRCA1 mutations were reported to be as 185delAG, 5382insC, and C61G. ${ }^{9}$ However, in our study, we could not detect common mutations except for BRCA1:c.5266dupC. The most common recurrent mutations were BRCA2:c.3318C $>\mathrm{G}$, BRCA2:c.632-2A > C, BRCA1:c.5266dupC, BRCA2:c.9317G > A, BRCA2:c.8452G > A in our study. More recurrent BRCA2 variants were observed when compared with BRCA1. Some studies indicate that those who carry the BRCA1 variant have an increased risk of developing cancer at an earlier age, but our study supports that BRCA2 mutation carriers could develop cancer at an earlier age. ${ }^{9}$ Mean and median age $(48,47$, respectively) of the patients with BRCA2 variants were lower than the mean and median age of the patients with BRCA1 variants $(49,48$, respectively). We observed high BRCA1-2 mutation frequency in triple-negative breast cancer patients, as mentioned in the literature. ${ }^{22}$

The results of our study help provide current information about the relationship between BRCA1 and BRCA2 mutation status and the development of some cancers. With advancements in next-generation sequencing (NGS), testing multiple genes has been both quicker and easier. The key strength of our research is that it included a broad, well-defined group of participants, all of whom had symptoms that appeared in the clinical context, making our results generalizable to patients. We used a specialized diagnostic center with considerable cancer gene testing expertise to conduct an exhaustive and practical cancer gene study. Patients were referred to our clinic from many different regions of Turkey. Although sometimes it was impossible to test all the family members at risk, a limitation we encountered, genetic counseling was done.

Our study gives a novel insight into the diagnosis of patients suspected of having LS. Testing with gene panels is essential to identify patients at a high risk of cancer. Our data demonstrate that BRCA12 mutations are associated with an increased risk for familial cancers and should be considered when ordering genetic testing for individuals with a personal or family history of cancer, particularly colorectal cancer. In any genetic cancer panel performed for a patient with a history suspected of Lynch syndrome or HBOC, the BRCA1-2 analysis should be considered. Further research could shed light on DNA repair genes' unique carcinogenesis roles. Large cohorts are needed to detect and clarify the critical founder mutations. A more thorough assessment of cancer genetics studies would be essential to strengthen patient risk assessment, prognosis, and treatment decisions.

\section{REFERENCES}

1. Howlader N, Noone A, Krapcho M, et al. SEER Cancer Statistics Review. National Cancer Institute 1975-2012, 2015 http://seer.cancer.gov/csr/1975_2012/, based on November 2014 SEER data submission, posted to the SEER web site. 
2. Nagy R, Sweet K, Eng C. Highly penetrant hereditary cancer syndromes. Oncogene 23: 6445-6470, 2004.

3. Southey MC, Goldgar DE, Winqvist R, et al. PALB2, CHEK2 and ATM rare variants and cancer risk: Data from COGS. J Med Genet 53: 800-811, 2016.

4. Valencia OM, Samuel SE, Viscusi RK, et al. The role of genetic testing in patients with breast cancer a review. JAMA Surg 152: 589-594, 2017.

5. Eccles BK, Copson E, Maishman T, et al. Understanding of BRCA VUS genetic results by breast cancer specialists. BMC Cancer 15: 936, 2015.

6. Judkins T, Rosenthal E, Arnell C, et al. Clinical significance of large rearrangements in BRCA1 and BRCA2. Cancer 118: 5210-5216, 2012.

7. Thompson D, Easton DF. Cancer incidence in BRCA1 mutation carriers. J Natl Cancer Inst 94: 1358-1365, 2002.

8. Cousineau I, Abaji C, Belmaaza A. BRCA1 regulates RAD51 function in response to DNA damage and suppresses spontaneous sister chromatid replication slippage: Implications for sister chromatid cohesion, genome stability, and carcinogenesis. Cancer Res 65: 11384-11391, 2005.

9. Mahdavi M, Nassiri M, Kooshyar MM, et al. Hereditary breast cancer; Genetic penetrance and current status with BRCA. J Cell Physiol 234: 5741-5750, 2019.

10. de Bono J, Ramanathan RK, Mina L, et al. Phase I, doseescalation, two-part trial of the PARP inhibitor talazoparib in patients with advanced germline BRCA1/2 mutations and selected sporadic cancers. Cancer Discov 7: 620-629, 2017.

11. Vinayak S, Ford JM. PARP inhibitors for the treatment and prevention of breast cancer. Curr Breast Cancer Rep 2: 190197, 2010.

12. Varqa AN. Natural history of ovarian cancer. Ecancermedicalscience 8: 465, 2014.

13. Golan T, Kanji ZS, Epelbaum R, et al. Overall survival and clinical characteristics of pancreatic cancer in BRCA mutation carriers. Br J Cancer 111: 1132-1138, 2014.

14. Beger C, Ramadani M, Meyer S, et al. Down-regulation of BRCA1 in chronite pancreatitis and sporadic pancreatic adenocarcinoma. Clin Cancer Res 10: 3780-3787, 2004.

15. Richards S, Aziz N, Bale S, et al. Standards and guidelines for the interpretation of sequence variants: $\mathrm{A}$ joint consensus recommendation of the American College of Medical Genetics and Genomics and the Association for Molecular Pathology. Genet Med 17: 405-424, 2015.
16. On M, McBride A, Yun S, et al. BRCA1 and BRCA2 gene mutations and colorectal cancer risk: Systematic review and meta-analysis. J Natl Cancer Inst 110: 1178-1189, 2018.

17. Livraghi L, Garber JE. PARP inhibitors in the management of breast cancer: Current data and future prospects. BMC Med 13: 188, 2015.

18. Daly MB, Pal T, Berry MP, et al. Genetic/familial high-risk assessment: Breast, ovarian, and pancreatic, version 2.2021. JNCCN J Natl Compr Cancer Netw 19: 77-102, 2021.

19. Hall MJ, Reid JE, Burbidge LA, et al. BRCA1 and BRCA2 mutations in women of different ethnicities undergoing testing for hereditary breast-ovarian cancer. Cancer 115: 2222-2233, 2009.

20. Nelson-Moseke AC, Jeter JM, Cui H, Roe DJ, Chambers SK, Laukaitis CM. An unusual BRCA mutation distribution in a high risk cancer genetics clinic. Fam Cancer 12: 83-87, 2013.

21. Thompson ER, Gorringe KL, Rowley SM, et al. Reevaluation of the BRCA2 truncating allele c.9976A >t (p.Lys3326Ter) in a familial breast cancer context. Sci Rep 5: 14800, 2015.

22. Armstrong N, Ryder S, Forbes $\mathrm{C}$, et al. A systematic review of the international prevalence of BRCA mutation in breast cancer. Clin Epidemiol 11: 543-561, 2019.

\section{Correspondence: \\ Dr. Ibrahim SAHIN}

Diskapi Yildirim Beyazit Egitim ve Arastirma Hastanesi,

Tibbi Genetik Bolumu

Diskapi, ANKARA / TURKEY

Tel: (+90-536) 8738442

Fax: (+90-312) 5962000

e-mail: ibrahimsahinmd@gmail.com

\section{ORCIDs:}

Ibrahim Sahin

0000-0002-6050-816X

Hanife Saat

0000-0002-6087-5947

Sercan Aksoy 